Check for updates

Cite this: RSC Adv., 2018, 8, 38166

Received 25th September 2018 Accepted 5th November 2018

DOI: 10.1039/c8ra07940d

rsc.li/rsc-advances

\section{Preparation of 3-hydroxyquinolines from direct oxidation of dihydroquinolinium salts $\uparrow$}

\author{
Mani Ramanathan, $\$$ Jing Wan $\$$ and Shiuh-Tzung Liu (DD* \\ A series of functionalized 3,4-dihydroquinolinium salts were prepared from the reaction of aryldiazonium \\ salt with alkene in a nitrile solution. Further oxidation yielding either 3-hydroxyquinoline or quinoline \\ products was investigated. A one-pot process from aryldiazonium salts, alkenes and nitriles leading to 3- \\ hydroxyquinolines was also developed. Furthermore, an intramolecular trapping of an $\mathrm{N}$-arylnitrilium ion \\ with a vinyl group at the ortho position leading to 2-substituted quinolines was revealed.
}

\section{Introduction}

Functionalized quinolines are highly privileged structural units due to their widespread occurrence in various natural products and functional materials. ${ }^{1}$ The quinoline core is known to be a versatile building block in the preparation of small molecules with diverse therapeutic properties. ${ }^{2}$ Among the various quinoline derivatives, 3-hydroxyquinolines (quinoline-3-ols) are privileged (Fig. 1). ${ }^{3}$ Typically, Talnetant is a potential neurokinin 3 receptor antagonist primarily used in irritable bowel syndrome and psychotic disorders, ${ }^{3 a}$ whereas PSI-697 is utilized as an orally active effective P-selectin inhibitor. ${ }^{3 b}$ These ubiquitous cores have also been studied as selective inhibitors HIV-1 reverse transcriptase, ${ }^{4}$ antiallergic agents (TA-270), ${ }^{5}$ antioxidant $^{3 c}$ and $\mathrm{D}$-amino acid oxidase inhibitors (DAAO). ${ }^{6}$ In addition, these frameworks have been used as building blocks in functional materials. ${ }^{7}$ Notably, naturally occurring anti-HIV fungal metabolites such as viridicatol, viridication and 3-Omethylviridicatin are derivatives of 3-hydroxyquinolin-2 $(1 H)$ ones. $^{8}$

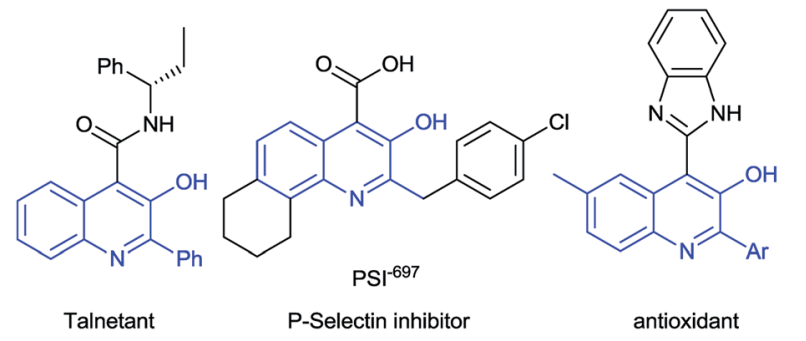

Fig. 1 Selected 3-hydroxyquinoline derivatives.

Department of Chemistry, National Taiwan University, Taipei, Taiwan 106. E-mail: stliu@ntu.edu.tw

$\dagger$ Electronic supplementary information (ESI) available: Spectral data of all compounds and spectra. See DOI: 10.1039/c8ra07940d

\$ Equal contribution.
Despite the excellent significance, synthetic efforts towards this ubiquitous core remain largely underdeveloped. Classical routes leading to 3-hydroxyquinoline derivatives are Friedlander type condensations, ${ }^{9}$ Pfitzinger synthesis, ${ }^{10}$ Danheiser type annulation of 2-iodostyrenes with acetylenes, ${ }^{11}$ Knoevenagel condensation followed by decyanative epoxide-arene cyclization, ${ }^{12}$ and Cromwell's approach of epoxide opening/ condensation of $o$-aminobenazalacetophenone oxides. ${ }^{13}$ In recent years, Jamison et al. demonstrated a convenient synthesis of 3-hydroxyquinolines via aerobic oxidation of corresponding aryl Grignard reagents under a continuous flow method..$^{14}$ In 2017, Yadav's group described a NBS mediated deacylative formation of 3-hydroxyquinolines from 3-acetylquinolines via an epoxide intermediate. ${ }^{15}$ More recently, Mamedov et al. developed an in situ Meinwald rearrangementintramolecular reductive cyclization of $o$-nitroarylacetophenone oxides under mild conditions. ${ }^{16}$ Nevertheless, the above-mentioned routes require pre-functionalized

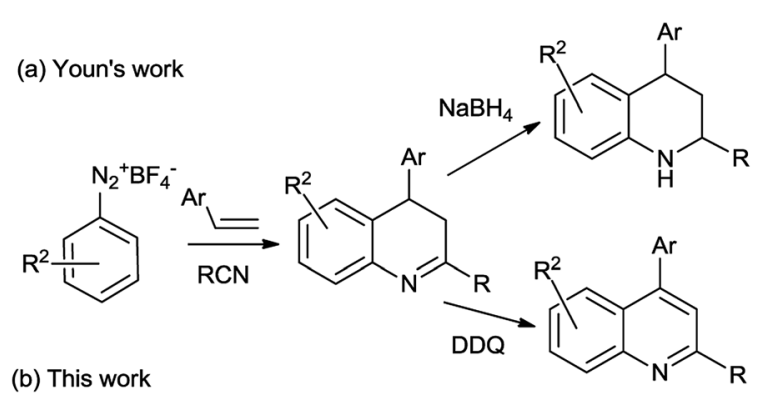

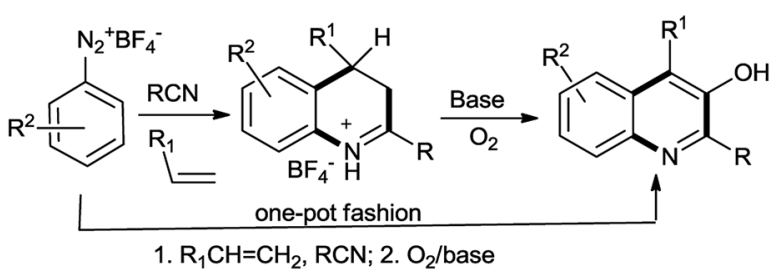

Scheme 1 Strategies leading to quinolone derivatives. 
substrates, metal catalysts and/or multistep synthetic protocol with a limited functionality presented in the molecule. Thus, there is still a need for the development of a convenient method from easily available precursors with a diversity of functionality.

In early this year, Youn's group reported the reaction of aryldiazonium salts with nitrile followed by the cyclization with alkenes to achieve 3,4-dihydroquinolines, which was subsequently either oxidized or reduced to yield the corresponding quinolines or tetrahydroquinolines, respectively (Scheme 1a). ${ }^{17}$ In this work, we adopted the similar method to prepare 3,4dihydroquinolines followed by a further oxidation at C-3 position leading to 3-hydroxyquinolines (Scheme 1b).

\section{Results and discussion}

\section{Preparation of dihydroquinolinium salts}

According to Youn's procedure, we have prepared a series of 4aryl-dihydroquinolines by reaction of aryldiazonium tetrafluoroborate with styrenes in a nitrile solution (Table 1). Interestingly, when arylnitrile was employed, the corresponding 2,4diaryl substituted quinolinium salt $(\mathbf{3 h})$ was obtained as the major products, but accompanied with quinazolines (4) as the side product (Scheme 2). ${ }^{18 c}$ Similarly, when $\alpha$-methylstyrene was used as the substrate, a 4,4-disubstituted dihydroquinoline $3 \mathrm{~m}$ was isolated in $49 \%$ yield. However, reaction of $\alpha$-bromostyrene with $\mathbf{1 a}$ in acetonitrile gave quinoline $\mathbf{5}$ as the single product presumably due the elimination of $\mathrm{HBr}$ (Scheme 3). Instead of styrene, norbornylene smoothly participated in the reaction leading to fused-tricyclic quinolinium salts $3 \mathbf{n}$ in $62 \%$ yield (eqn. (1)).

Table 1 Isolation of dihydroquinolines ${ }^{a}$
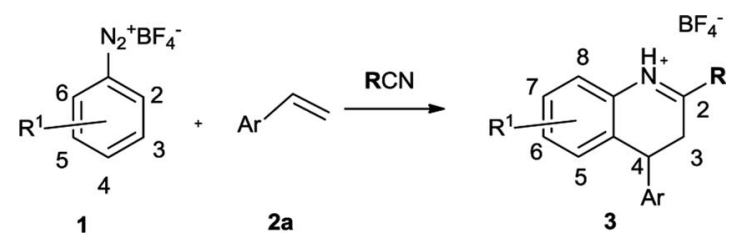

\begin{tabular}{lll} 
Entry & Substituents & Product 3 (yield) \\
\hline 1 & $\mathrm{R}^{1}=\mathrm{H}, \mathrm{Ar}=\mathrm{Ph}, \mathrm{R}=\mathrm{Me}$ & $\mathbf{3 a},(81 \%)$ \\
2 & $\mathrm{R}^{1}=4-\mathrm{Me}, \mathrm{Ar}=\mathrm{Ph}, \mathrm{R}=\mathrm{Me}$ & $3 \mathbf{b}, \mathrm{R}^{1}=6-\mathrm{Me}(72 \%)$ \\
3 & $\mathrm{R}^{1}=4-\mathrm{Me}, \mathrm{Ar}=\mathrm{Ph}, \mathrm{R}=\mathrm{Ph}$ & $\mathbf{3 c}, \mathrm{R}^{1}=6-\mathrm{Me}(54 \%)$ \\
4 & $\mathrm{R}^{1}=4-(t-\mathrm{Bu}), \mathrm{Ar}=\mathrm{Ph}, \mathrm{R}=\mathrm{Me}$ & $\mathbf{3 d}, \mathrm{R}^{1}=6 t-\mathrm{Bu}(68 \%)$ \\
5 & $\mathrm{R}^{1}=\mathrm{H}, \mathrm{Ar}=\mathrm{Ph}, \mathrm{R}=\mathrm{Pr}$ & $\mathbf{3 e},(63 \%)$ \\
6 & $\mathrm{R}^{1}=\mathrm{H}, \mathrm{Ar}=\mathrm{Ph}, \mathrm{R}=\mathrm{i}-\mathrm{Pr}$ & $\mathbf{3 f},(57 \%)$ \\
7 & $\mathrm{R}^{1}=\mathrm{H}, \mathrm{Ar}=\mathrm{Ph}, \mathrm{R}=\mathrm{c}-\mathrm{C}_{3} \mathrm{H}_{5}$ & $\mathbf{3 g},(66 \%)$ \\
8 & $\mathrm{R}^{1}=\mathrm{H}, \mathrm{Ar}=\mathrm{Ph}, \mathrm{R}=\mathrm{Ph}$ & $\mathbf{3 h},(67 \%)^{b}$ \\
9 & $\mathrm{R}^{1}=\mathrm{H}, \mathrm{Ar}=p-\mathrm{BrC}_{6} \mathrm{H}_{4}, \mathrm{R}=\mathrm{Me}$ & $\mathbf{3 i},(80 \%)$ \\
10 & $\mathrm{R}^{1}=\mathrm{H}, \mathrm{Ar}=p-\mathrm{ClC}_{6} \mathrm{H}_{4}, \mathrm{R}=\mathrm{Me}$ & $\mathbf{3 j},(62 \%)$ \\
11 & $\mathrm{R}^{1}=\mathrm{H}, \mathrm{Ar}=p-\mathrm{ClCH}_{2} \mathrm{C}_{6} \mathrm{H}_{4}, \mathrm{R}=\mathrm{Me}$ & $\mathbf{3 k},(76 \%)$ \\
12 & $\mathrm{R}^{1}=\mathrm{H}, \mathrm{Ar}=2,5-\mathrm{Me}_{2} \mathrm{C}_{6} \mathrm{H}_{3}, \mathrm{R}=\mathrm{i}-\mathrm{Pr}$ & $\mathbf{3 l},(39 \%)$
\end{tabular}

${ }^{a}$ Reaction conditions: a mixture of $1 \mathrm{a}(0.52 \mathrm{mmol}), 2 \mathrm{a}(1.04 \mathrm{mmol})$ and solvent $(2 \mathrm{~mL})$ in reaction tube. ${ }^{b}$ Quinazoline $\mathbf{4}$ as the side product $(18 \%)$.

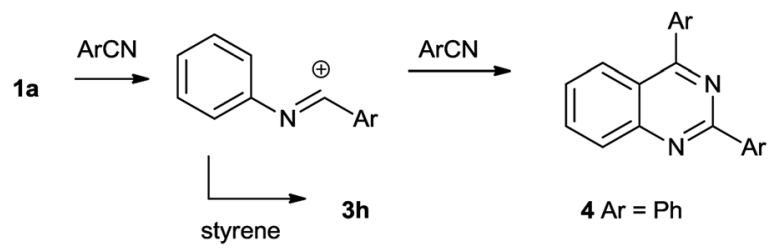

Scheme 2 Reactions of 1a with benzonitrile.

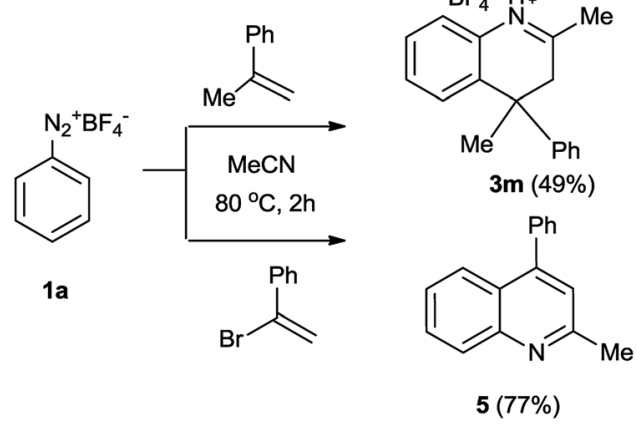

Scheme 3 Reactions of 1a with 1,1-disubstituted styrenes.
$1 \mathrm{a}$

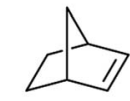

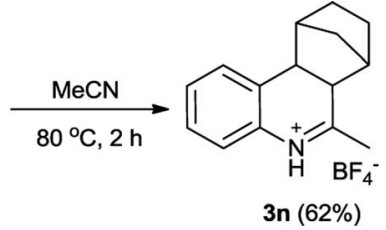

(1)

\section{Oxidization of 3,4-dihydroquinolinium salts}

During the chromatographic purification of dihydroquinoline, we learned that both quinoline and 3-hydroxyquinoline, resulting from the oxidation, were obtained. This observation raised our attention of the preparation of 3-hydroxyquinolines from dihydroquinoline via oxidation. Thus, further studies focused on this issue with the use of 3a as a model. Basically, we proposed that the allylic oxidation giving $\mathbf{6} \mathbf{a}^{\prime}$ followed by tautomerization should render the desired product (Scheme 4). Table 2 summarizes the results of the oxidation of $\mathbf{3 a}$ under various conditions.

Quite a lot of procedures were attempted in the oxidation of $\mathbf{3 a}$, but in the most cases leading the quinoline $\mathbf{5}$, to be a favoured process. Fortunately, the desired hydroxyquinoline $\mathbf{6 a}$<smiles>CC1=Nc2ccc(C(F)(F)F)cc2C(c2ccccc2)C(=O)C1(C)C(C)(C)C(C)(C)C</smiles>

Scheme 4 Oxidation of dihydroquinoline $3 a$ leading to 3 hydroxyquinoline. 
Table 2 Optimization for oxidation of $3 a$ into $6 a$ and $5^{a}$<smiles>Cc1cc(-c2ccccc2)c2ccccc2n1</smiles>

\begin{tabular}{|c|c|c|c|}
\hline atry & Conditions & $6 a$ & 5 \\
\hline & $\mathrm{H}_{2} \mathrm{O}_{2}$ (2 eq. $/ / \mathrm{CH}_{3} \mathrm{CN}$ & - & - \\
\hline & $\mathrm{H}_{2} \mathrm{O}_{2}$ (2 eq. $) / \mathrm{Na}_{2} \mathrm{CO}_{3} / \mathrm{CH}_{3} \mathrm{CN}$ & $30 \%$ & $55 \%$ \\
\hline & Oxone (1 eq.) $/ \mathrm{CH}_{3} \mathrm{CN}$ & $10 \%$ & $58 \%$ \\
\hline & Oxone (1 eq.) $/ \mathrm{Na}_{2} \mathrm{CO}_{3}$ (1 eq.) $/ \mathrm{CH}_{3} \mathrm{CN}$ & $30 \%$ & $43^{\circ}$ \\
\hline & Silica gel $/ \mathrm{NaHCO}_{3} / \mathrm{CH}_{3} \mathrm{CN}$ & $15 \%$ & $42^{\circ}$ \\
\hline & $\mathrm{I}_{2}$ (1 eq.) $/ \mathrm{Na}_{2} \mathrm{CO}_{3}$ (1 eq.) $/ \mathrm{CH}_{3} \mathrm{CN}$ & $15 \%$ & 770 \\
\hline & $\mathrm{O}_{2}(1 \mathrm{~atm}) / \mathrm{Na}_{2} \mathrm{CO}_{3}$ (1 eq. $) / \mathrm{CH}_{3} \mathrm{CN} / 50^{\circ} \mathrm{C}$ & $14 \%$ & $30^{\circ}$ \\
\hline & $\mathrm{TBHP}(2$ eq. $) / \mathrm{O}_{2}(1 \mathrm{~atm}) / \mathrm{CH}_{3} \mathrm{CN}$ & $22 \%$ & 33 \\
\hline & $\mathrm{S}_{8}(1$ eq. $) / \mathrm{N}_{2}(1 \mathrm{~atm}) / \mathrm{CH}_{3} \mathrm{CN}$ & - & $76^{\circ}$ \\
\hline & $\mathrm{Cu}_{2} \mathrm{O}(1 \mathrm{eq}.) / \mathrm{O}_{2}(1 \mathrm{~atm}) / \mathrm{CH}_{3} \mathrm{CN}$ & $26 \%$ & 37 \\
\hline & $\mathrm{Cu}(\mathrm{OAc})_{2}(1 \mathrm{eq}.) / \mathrm{O}_{2}(1 \mathrm{~atm}) / \mathrm{CH}_{3} \mathrm{CN}$ & $8 \%$ & 56 \\
\hline & $\mathrm{Na}_{2} \mathrm{WO}_{4}(0.1 \mathrm{mmol}) / \mathrm{H}_{2} \mathrm{O}_{2}(1.2$ eq. $) / \mathrm{CH}_{3} \mathrm{CN} / 2 \mathrm{~h}$ & $6 \%$ & 36 \\
\hline & $\operatorname{TBHP}(1.2$ eq. $) / \mathrm{VO}(\mathrm{acac})_{2}(0.1 \mathrm{mmol}) / \mathrm{CH}_{3} \mathrm{CN} / 2 \mathrm{~h}$ & $30 \%$ & 70 \\
\hline & AIBN (1 eq.) $/ \mathrm{CH}_{3} \mathrm{CN} / \mathrm{N}_{2} /$ reflux & - & 93 \\
\hline & $\mathrm{KOH}(2$ eq. $) / \mathrm{O}_{2}(1 \mathrm{~atm}) / \mathrm{CH}_{3} \mathrm{CN}$ & $6 \%$ & $61^{\circ}$ \\
\hline & $\mathrm{Na}_{2} \mathrm{CO}_{3}$ (1 eq. $) / \mathrm{O}_{2}(1 \mathrm{~atm}) / \mathrm{CH}_{3} \mathrm{CN}$ & $11 \%$ & 51 \\
\hline & $\mathrm{Na}_{2} \mathrm{CO}_{3}(10$ eq. $) / \mathrm{O}_{2}(1 \mathrm{~atm}) / \mathrm{CH}_{2} \mathrm{Cl}_{2} / 72 \mathrm{~h}$ & $73 \%$ & $25^{c}$ \\
\hline & $\mathrm{Na}_{2} \mathrm{CO}_{3}(10$ eq. $) / \mathrm{O}_{2}(1 \mathrm{~atm}) / \mathrm{MeOH} / 72 \mathrm{~h}$ & $12 \%$ & $60^{\circ}$ \\
\hline & $\mathrm{Na}_{2} \mathrm{CO}_{3}$ (10 eq.)/TEMPO/O $\left./ 1 \mathrm{~atm}\right) / \mathrm{CH}_{2} \mathrm{Cl}_{2} / 72 \mathrm{~h}$ & $18 \%$ & \\
\hline
\end{tabular}

${ }^{a}$ Reaction conditions: a mixture of $3 \mathbf{a}(0.16 \mathrm{mmol})$ in solvent $(2 \mathrm{~mL})$ was treated with oxidant as indicated in each entry.

was obtained in $73 \%$ yield upon treating with $\mathrm{O}_{2}$ in the presence of excess of $\mathrm{Na}_{2} \mathrm{CO}_{3}$ accompanied with $25 \%$ of $\mathbf{5}$ (Table 2, entry 17). By addition of an equimolar amount of $(2,2,6,6-$ tetramethylpiperidin-1-yl)oxyl (TEMPO), the reaction was inhibited dramatically, indicating that a possible radical pathway is involved in this oxidation (Table 2, entry 19). Thus, a rationalization of formation of $\mathbf{5}$ versus $\mathbf{6 a}$ is summarized in Scheme 5 . The abstraction of hydrogen atoms from benzylic or allylic positions would lead to the products. In particular, the reaction of $\mathrm{O}_{2}$ with intermediate $3 \mathbf{a}^{\prime \prime}$ yields the desired product 6a.

Few dihydroquinolines were then transformed into the corresponding 3-hydroxyquinolines smoothly by adopting the

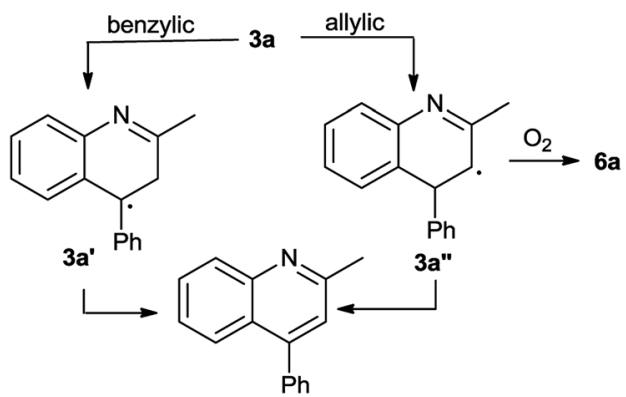

Scheme 5 Abstraction of hydrogen from benzylic versus allylic positions.<smiles>[R]C1=[NH+]c2ccc([R])cc2C([R])C1</smiles><smiles>[R]c1ccc2nc([R])c(O)c([R])c2c1</smiles>

6a, $\mathrm{R}^{1}=\mathrm{H}, \mathrm{R}^{2}=\mathrm{Ph}, \mathrm{R}^{3}=\mathrm{Me}(73 \%)$

6b, $R^{1}=H, R^{2}=p-C_{C} \mathrm{H}_{2} \mathrm{C}_{6} \mathrm{H}_{4}, \mathrm{R}^{3}=\mathrm{Me}(69 \%)$

6c, $\mathrm{R}^{1}=\mathrm{H}, \mathrm{R}^{2}=\mathrm{p}-\mathrm{ClC}_{6} \mathrm{H}_{4}, \mathrm{R}^{3}=\mathrm{Me}(50 \%)$

6d, $R^{1}=M e, R^{2}=P h, R^{3}=M e(53 \%)$

6h, $\mathrm{R}^{1}=\mathrm{H}, \mathrm{R}^{2}=\mathrm{Ph}, \mathrm{R}^{3}=\mathrm{c}-\operatorname{Pr}(55 \%)$

$6 j, R^{1}=H, R^{2}=P h, R^{3}=P h(54 \%)$

6k, $\mathrm{R}^{1}=\mathrm{H}, \mathrm{R}^{2}=\mathrm{Ph}, \mathrm{R}^{3}=t-\mathrm{Bu}(0 \%)$

Scheme 6 Oxidation of dihydroquinlines to give 3-hydroxyquinolines (NMR yields).

same procedure (Scheme 6). In case of $3 \mathbf{m}$, the oxidation gave 4methyl-4-phenylquinolin-3(4H)-one (7) (eqn (2)), ${ }^{19}$ which is in agreement of the proposed pathway of this oxidation in Scheme 4.

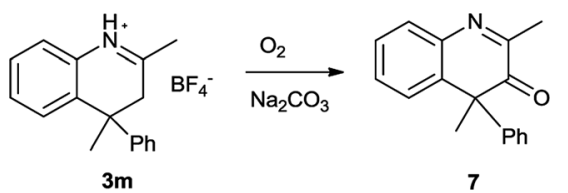

\section{One-pot fashion of preparation of 3-hydoxyquinolines}

We attempted to carry out the two step reactions in a one-pot fashion (Table 3). Various aryldiazonium salts, nitriles and alkenes participated well in this oxidative transformation to afford structurally multi-substituted 3-hydroxyquinoline frameworks. Typically, phenyldiazonium tetrafluoroborate was treated with styrene in anhydrous MeCN under conditions identical to those employed previously (Table 1), followed by treatment of $\mathrm{Na}_{2} \mathrm{CO}_{3}$ and prolonged exposure to $\mathrm{O}_{2}$ furnished

Table 3 Preparation of 3-hydroxyquinolines in one-pot fashion ${ }^{a}$

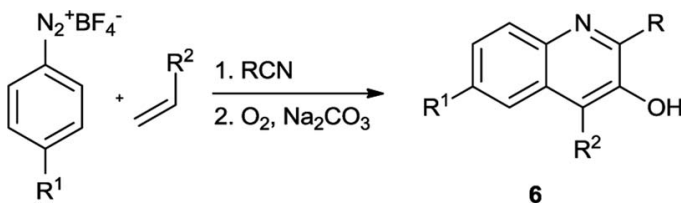

\begin{tabular}{lll}
\hline Entry & Substituents in $\mathbf{6}$ & Product (yield) \\
\hline 1 & $\mathrm{R}=\mathrm{Me}, \mathrm{R}^{1}=\mathrm{H}, \mathrm{R}^{2}=\mathrm{C}_{6} \mathrm{H}_{5}$ & $\mathbf{6 a}(36 \%)$ \\
2 & $\mathrm{R}=\mathrm{Me}, \mathrm{R}^{1}=\mathrm{H}, \mathrm{R}^{2}=p-\left(\mathrm{ClCH}_{2}\right) \mathrm{C}_{6} \mathrm{H}_{4}$ & $\mathbf{6 b}(34 \%)$ \\
3 & $\mathrm{R}=\mathrm{Me}, \mathrm{R}^{1}=\mathrm{Me}, \mathrm{R}^{2}=\mathrm{C}_{6} \mathrm{H}_{5}$, & $\mathbf{6 d}(47 \%)$ \\
4 & $\mathrm{R}=\mathrm{Me}, \mathrm{R}^{1}=t-\mathrm{Bu}, \mathrm{R}^{2}=\mathrm{C}_{6} \mathrm{H}_{5}$ & $\mathbf{6 e ~ ( 4 4 \% )}$ \\
5 & $\mathrm{R}=n-\mathrm{Pr}, \mathrm{R}^{1}=\mathrm{H}, \mathrm{R}^{2}=\mathrm{C}_{6} \mathrm{H}_{5}$, & $\mathbf{6 f}(32 \%)$ \\
6 & $\mathrm{R}=\mathrm{i}-\mathrm{Pr}, \mathrm{R}^{1}=\mathrm{H}, \mathrm{R}^{2}=\mathrm{C}_{6} \mathrm{H}_{5}$ & $\mathbf{6 g}(39 \%)$ \\
7 & $\mathrm{R}=\mathrm{c}-\mathrm{Pr}, \mathrm{R}^{1}=\mathrm{H}, \mathrm{R}^{2}=\mathrm{C}_{6} \mathrm{H}_{5}$ & $\mathbf{6 h}(42 \%)$ \\
8 & $\mathrm{R}=\mathrm{Me}, \mathrm{R}^{1}=\mathrm{H}, \mathrm{R}^{2}=\mathrm{CH}_{3}\left(\mathrm{CH}_{2}\right)_{5}$ & $\mathbf{6 i}(56 \%)$ \\
9 & $\mathrm{R}=\mathrm{C}_{6} \mathrm{H}_{5}, \mathrm{R}^{1}=\mathrm{H}, \mathrm{R}^{2}=\mathrm{C}_{6} \mathrm{H}_{5}$ & $\mathbf{6 j}(46 \%)$
\end{tabular}

${ }^{a}$ Reaction conditions: a mixture of arenediazonium salt $(0.52 \mathrm{mmol})$, alkene $(1.04 \mathrm{mmol})$ and nitrile $(2 \mathrm{~mL})$ in reaction tube at $80{ }^{\circ} \mathrm{C}$ for $2 \mathrm{~h}$; followed by an aqueous workup with saturated aq. $\mathrm{NaHCO}_{3}$ and stirred in $\mathrm{O}_{2}(1 \mathrm{~atm})$ with $5 \mathrm{~g}$ of $\mathrm{Na}_{2} \mathrm{CO}_{3}$ for 3 days. 
<smiles>O=[N+]([O-])c1ccccc1-c1ccccc1</smiles>

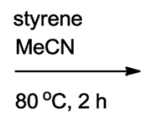<smiles>Cc1nc2ccccc2c2ccccc12</smiles>
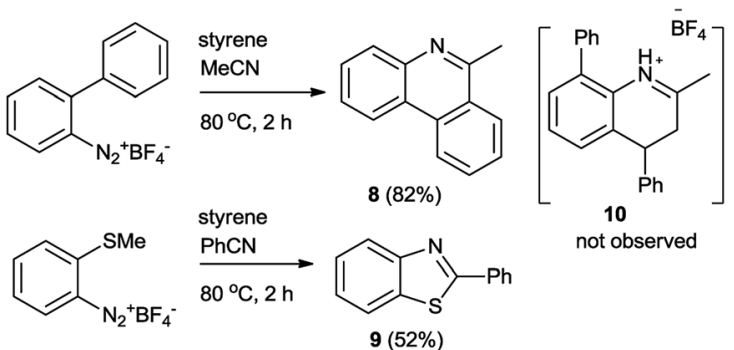

Scheme 7 Intra- versus inter-molecular reactions.

the 3-hydroxyquinoline derivative $6 \mathbf{a}$ in $36 \%$ isolated yield (Table 3, entry 1). Aryldiazonium salts bearing electrondonating substituents (Me-, $t$-Bu-) afforded the corresponding 3-hydroxyquinolines in acceptable yields (Table 3, entries 3-4). It should be noted that minor amount of 2,4-disubstituted quinoline derivatives were also isolated from the reaction mixture. Several aliphatic nitriles such as, primary, secondary, cyclic nitriles involved in the reaction and smoothly delivered the corresponding products (Table 3, entries 5-7). Unfortunately, benzylnitrile and chloroacetonitrile failed to produce the corresponding adducts. To our delight, aliphatic alkene was also a viable substrate for this oxidative transformation and provided the corresponding 2,4-dialkyl-3-hydroxyquinoline in good yield (Table 3, entry 8). As expected, benzonitrile furnished the expected adduct (6j) together with $21 \%$ of 2,4 phenylquinazoline.

When aryldiazonium salt bearing adjacent phenyl or methylthio moiety was employed, $N$-arylnitrilium intermediate expeditiously underwent the intramolecular cyclization to deliver 6-methyl-phenanthridine ${ }^{\mathbf{1 8}}(\mathbf{8})$ and 2-substituted benzothiazole (9) (Scheme 7). Presumably, the intra-molecular pathway is superior to the inter-molecular one.

Besides the intermolecular reaction with styrenes, we also examined the reaction via an intramolecular fashion. Thus, $o$ ethenylbenzenediazonium salt $\mathbf{1 1}$ was used for investigation. Reaction of 11 with various nitriles gave the corresponding 2substituted quinolines 12a-d in good yields (eqn (3)). Obviously, the nucleophilic attack of $\mathrm{C}=\mathrm{C}$ toward nitrilium ion followed by elimination took place immediately to deliver the final product.<smiles>C=Cc1ccccc1N=[N+]=[I-]</smiles>
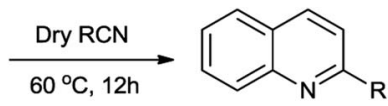

11 12a $\mathrm{R}=\mathrm{CH}_{3}(74 \%)$

12b $R=\operatorname{Pr}(69 \%)$

12c $R=i-\operatorname{Pr}(63 \%)$

12d $R=\operatorname{Ph}(69 \%)$

\section{Conclusions}

In summary, we have demonstrated a synthetic useful approach for the preparation of 3-hydroxyquinolines from dihydroquinolines under atmospheric pressure of oxygen in the presence of sodium carbonate. This preparation can be carried out from diazonium salts in a one-pot fashion. Intramolecular trapping of in situ generated $\mathrm{N}$-arylnitrilium intermediate with an alkene could generate 2-substituted quinolines in good yields. Preparation of functionalized 3-hydroxyquinoline derivatives through other known synthetic avenues generally requires a multistep synthesis.

\section{Experimental}

${ }^{1} \mathrm{H}$ and ${ }^{13} \mathrm{C}$ NMR were recorded in a $400 \mathrm{MHz}$ Bruker spectrometer. Chemical shifts were given relative to $\mathrm{CDCl}_{3}, \mathrm{CD}_{3} \mathrm{CN}$ and $\mathrm{DMSO}_{\mathrm{d}}$. Acetonitrile and other liquid nitriles were dried over activated molecular sieves, respectively, whereas solid nitriles were dried under vacuum. All the anilines were commercially purchased and used for diazotization without further purification. Other chemicals were used as purchased. Flash chromatography was performed using silica gel 230-400 mesh. Analytical thin layer chromatography (TLC) was performed using Merck-60 F-254 plates. In cases of known compounds, their ${ }^{1} \mathrm{H}$ and ${ }^{13} \mathrm{C}$ NMR values were compared with the literature values. Melting points were determined on a Fargo MP-1D instrument. All spectral data are deposited in the ESI. $\dagger$

\section{General procedure for preparation of 3,4-dihydroquinolines}

In a dry $10 \mathrm{~mL}$ glass sealed tube, phenyldiazonium salt $(100 \mathrm{mg}$, $0.52 \mathrm{mmol}, 1.0$ eq.) and styrene (108 mg, $1.04 \mathrm{mmol}, 2.0 \mathrm{eq}$. were suspended in $2 \mathrm{~mL}$ of anhydrous nitrile. The tube was sealed with a Teflon screw cap and heated in an oil bath $\left(80^{\circ} \mathrm{C}\right)$ for $2 \mathrm{~h}$. After cooling to room temperature, reaction mixture with diethyl ether $(20 \mathrm{~mL})$. Corresponding 3,4-dihydroquinolinium tetrafluoroborate salt was formed as a solid which was washed with $2 \times 10 \mathrm{~mL}$ of diethyl ether and dried under vacuum.

\section{2-Methyl-4-phenyl-3,4-dihydroquinolinium tetrafluoroborate $(3 a)^{17}$}

Off-white solid (130 mg, 81\%); mp 193-194 ${ }^{\circ} \mathrm{C}:{ }^{1} \mathrm{H}$ NMR (400 $\mathrm{MHz}, \mathrm{CDCl}_{3}$ ) $\delta 12.4$ (brs, $\left.1 \mathrm{H}\right), 7.52$ (dd, $\left.J=7.6,1.6 \mathrm{~Hz}, 1 \mathrm{H}\right), 7.48^{-}$ $7.44(\mathrm{~m}, 1 \mathrm{H}), 7.44-7.40(\mathrm{~m}, 2 \mathrm{H}), 7.39-7.32(\mathrm{~m}, 2 \mathrm{H}), 7.23-7.20$ $(\mathrm{m}, 2 \mathrm{H}), 7.03-7.00(\mathrm{~m}, 1 \mathrm{H}), 4.49(\mathrm{t}, J=8.8 \mathrm{~Hz}, 1 \mathrm{H}), 3.48-3.32(\mathrm{~m}$, 2H), $2.66(\mathrm{~s}, 3 \mathrm{H}) ;{ }^{13} \mathrm{C} \mathrm{NMR}\left(100 \mathrm{MHz}, \mathrm{CDCl}_{3}\right) \delta$ 189.2, 141.8, 133.2, 132.6, 131.3, 130.5, 130.3, 130.1, 129.2, 129.1, 121.8, 38.7,

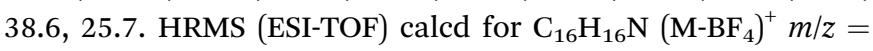
222.1277 , found 222.1274 . These data are in agreement with the reported data.

\section{2,6-Dimethyl-4-phenyl-3,4-dihydroquinolinium tetrafluoro- borate $(3 \mathbf{b})$}

Pale yellow solid (121 mg, 72\%). Mp 191-192 ${ }^{\circ} \mathrm{C} .{ }^{1} \mathrm{H}$ NMR (400 $\mathrm{MHz}, \mathrm{CD}_{3} \mathrm{CN}$ ): $\delta=12.41$ (brs, $\left.1 \mathrm{H}\right), 7.41-7.37$ (m, 3H), 7.35-7.31 $(\mathrm{m}, 1 \mathrm{H}), 7.27(\mathrm{~d}, J=8.0 \mathrm{~Hz}, 1 \mathrm{H}), 7.20(\mathrm{~d}, J=7.2 \mathrm{~Hz}, 2 \mathrm{H}), 6.85(\mathrm{~s}$, $1 \mathrm{H}), 4.43(\mathrm{t}, J=8.8 \mathrm{~Hz}, 1 \mathrm{H}), 3.44-3.28(\mathrm{~m}, 2 \mathrm{H}), 2.63(\mathrm{~s}, 3 \mathrm{H}), 2.27$ (s, 3H) ppm. ${ }^{13} \mathrm{C}$ NMR (100 MHz, $\left.\mathrm{CD}_{3} \mathrm{CN}\right): \delta=187.3,143.3$, 141.8, 130.9, 130.8, 130.6, 130.2, 129.0 (2C), 128.9, 121.4, 38.5, 38.4, 25.3, 21.5 ppm. HRMS (ESI-TOF) calcd for $\mathrm{C}_{17} \mathrm{H}_{18} \mathrm{~N}(\mathrm{M}-$ $\left.\mathrm{BF}_{4}\right)^{+} m / z=236.1434$, found 236.1443 . 
6-Methyl-2,4-diphenyl-3,4-dihydroquinolinium tetrafluoroborate $(3 \mathrm{c})$

Bright yellow solid (98 mg, 54\%). Mp 110-111 ${ }^{\circ} \mathrm{C} .{ }^{1} \mathrm{H}$ NMR (400 $\left.\mathrm{MHz}, \mathrm{CD}_{3} \mathrm{CN}\right): \delta=12.28$ (brs, $\left.1 \mathrm{H}\right), 8.07-8.05(\mathrm{~m}, 2 \mathrm{H}), 7.88-7.84$ $(\mathrm{m}, 1 \mathrm{H}), 7.72-7.65(\mathrm{~m}, 3 \mathrm{H}), 7.41-7.31(\mathrm{~m}, 4 \mathrm{H}), 7.27-7.24(\mathrm{~m}$, $2 \mathrm{H}), 4.61(\mathrm{t}, J=8.4 \mathrm{~Hz}, 1 \mathrm{H}), 3.92-3.79(\mathrm{~m}, 2 \mathrm{H}), 2.33(\mathrm{~s}, 3 \mathrm{H}) \mathrm{ppm}$. ${ }^{13} \mathrm{C}$ NMR $\left(100 \mathrm{MHz}, \mathrm{CD}_{3} \mathrm{CN}\right): \delta=177.5,143.8,141.3,137.7$, 131.8, 131.5, 131.0, 130.9 (2C), 130.5, 130.4, 130.2, 128.9 (2C), 122.5, 38.8, 36.0, 21.6 ppm. HRMS (ESI-TOF) calcd for $\mathrm{C}_{22} \mathrm{H}_{20} \mathrm{~N}$ $\left(\mathrm{M}-\mathrm{BF}_{4}\right)^{+} \mathrm{m} / z=298.1590$, found 298.1571 .

\section{6-(tert-Butyl)-2-methyl-4-phenyl-3,4-dihydroquinolinium tetrafluoroborate (3d)}

Pale yellow solid (101 mg, 68\%). Mp 190-191 ${ }^{\circ} \mathrm{C} .{ }^{1} \mathrm{H}$ NMR (400 $\mathrm{MHz}, \mathrm{CD}_{3} \mathrm{CN}$ ): $\delta=12.4$ (brs, $\left.1 \mathrm{H}\right), 7.51(\mathrm{dd}, J=8.4,1.6 \mathrm{~Hz}, 1 \mathrm{H})$, $7.46(\mathrm{~d}, J=8.4 \mathrm{~Hz}, 1 \mathrm{H}), 7.40-7.37(\mathrm{~m}, 2 \mathrm{H}), 7.34-7.30(\mathrm{~m}, 1 \mathrm{H})$, $7.20(\mathrm{~d}, J=7.2 \mathrm{~Hz}, 2 \mathrm{H}), 7.13(\mathrm{~d}, J=1.2 \mathrm{~Hz}, 1 \mathrm{H}), 4.50(\mathrm{t}, J=$ $8.4 \mathrm{~Hz}, 1 \mathrm{H}), 3.47-3.31(\mathrm{~m}, 2 \mathrm{H}), 2.64(\mathrm{~s}, 3 \mathrm{H}), 1.21(\mathrm{~s}, 9 \mathrm{H}) \mathrm{ppm} .{ }^{13} \mathrm{C}$ NMR (100 MHz, $\mathrm{CD}_{3} \mathrm{CN}$ ): $\delta=187.7,156.0,141.9,130.7,130.6$, 130.2, 128.9, 128.8, 127.3, 126.8, 121.3, 38.7, 38.5, 35.8, 31.2, 25.4 ppm. HRMS (ESI-TOF) calcd for $\mathrm{C}_{20} \mathrm{H}_{24} \mathrm{~N}\left(\mathrm{M}-\mathrm{BF}_{4}\right)^{+} \mathrm{m} / z=$ 278.1903, found 278.1914.

\section{4-Phenyl-2-propyl-3,4-dihydroquinolinium tetrafluoroborate} (3e)

Pale yellow solid (110 mg, 63\%). Mp 132-133 ${ }^{\circ} \mathrm{C} .{ }^{1} \mathrm{H}$ NMR (400 $\left.\mathrm{MHz}, \mathrm{CD}_{3} \mathrm{CN}\right): \delta=12.40$ (brs, $\left.1 \mathrm{H}\right), 7.59$ (d, $\left.J=7.6 \mathrm{~Hz}, 1 \mathrm{H}\right), 7.51-$ $7.44(\mathrm{~m}, 2 \mathrm{H}), 7.40-7.30(\mathrm{~m}, 3 \mathrm{H}), 7.17$ (d, $J=7.2 \mathrm{~Hz}, 2 \mathrm{H}), 7.08$ (d, $J$ $=7.2 \mathrm{~Hz}, 1 \mathrm{H}), 4.52(\mathrm{t}, J=8.0 \mathrm{~Hz}, 1 \mathrm{H}), 3.49-3.36(\mathrm{~m}, 2 \mathrm{H}), 2.93-$ $2.79(\mathrm{~m}, 2 \mathrm{H}), 1.77-1.58(\mathrm{~m}, 2 \mathrm{H}), 0.92(\mathrm{t}, J=7.6 \mathrm{~Hz}, 3 \mathrm{H}) \mathrm{ppm} .{ }^{13} \mathrm{C}$ NMR (100 MHz, $\left.\mathrm{CD}_{3} \mathrm{CN}\right): \delta=191.7,141.6,133.3,132.8,131.4$, 130.5, 130.4, 130.2, 129.1 (2C), 122.1, 40.9, 38.5, 37.5, 20.2, 14.0 ppm. HRMS (ESI-TOF) calcd for $\mathrm{C}_{18} \mathrm{H}_{20} \mathrm{~N}\left(\mathrm{M}-\mathrm{BF}_{4}\right)^{+} \mathrm{m} / z=$ 250.1590 , found 250.1593 .

\section{2-Isopropyl-4-phenyl-3,4-dihydroquinolinium} tetrafluoroborate (3f)

Pale yellow solid (101 mg, 57\%). Mp 148-149 ${ }^{\circ} \mathrm{C} .{ }^{1} \mathrm{H}$ NMR (400 $\left.\mathrm{MHz}, \mathrm{CD}_{3} \mathrm{CN}\right): \delta=12.20$ (brs, $\left.1 \mathrm{H}\right), 7.66(\mathrm{dd}, J=7.2,1.2 \mathrm{~Hz}, 1 \mathrm{H})$, 7.53-7.45 (m, 2H), 7.39-7.30 (m, 3H), 7.16-7.10 (m, 3H), $4.53(\mathrm{t}, J$ $=8.0 \mathrm{~Hz}, 1 \mathrm{H}), 3.47-3.35(\mathrm{~m}, 2 \mathrm{H}), 3.17$ (sept, $6.8 \mathrm{~Hz}, 1 \mathrm{H}), 1.27(\mathrm{~d}$, $J=6.8 \mathrm{~Hz}, 3 \mathrm{H}), 1.15(\mathrm{~d}, J=6.8 \mathrm{~Hz}, 3 \mathrm{H}) \mathrm{ppm} .{ }^{13} \mathrm{C} \mathrm{NMR}(100 \mathrm{MHz}$, $\left.\mathrm{CD}_{3} \mathrm{CN}\right): \delta=194.9,141.4,133.4,132.8,131.6,130.4,130.3$, 130.2, 129.1, 129.0, 122.4, 38.5, 35.2, 18.6, 18.5 ppm. HRMS (ESI-TOF) calcd for $\mathrm{C}_{18} \mathrm{H}_{20} \mathrm{~N}\left(\mathrm{M}-\mathrm{BF}_{4}\right)^{+} \mathrm{m} / z=250.1590$, found 250.1597.

\section{2-Cyclopropyl-4-phenyl-3,4-dihydroquinolinium} tetrafluoroborate $(3 g)$

Bright yellow solid (114 mg, 66\%). Mp 157-158 ${ }^{\circ} \mathrm{C} .{ }^{1} \mathrm{H}$ NMR (400 $\mathrm{MHz}, \mathrm{CD}_{3} \mathrm{CN}$ ): $\delta=12.3$ (brs, $\left.1 \mathrm{H}\right), 7.52-7.45$ (m, 2H), 7.41-7.31 $(\mathrm{m}, 4 \mathrm{H}), 7.17-7.14(\mathrm{~m}, 2 \mathrm{H}), 7.03(\mathrm{~d}, J=7.6 \mathrm{~Hz}, 1 \mathrm{H}), 4.42(\mathrm{t}, J=$ $8.0 \mathrm{~Hz}, 1 \mathrm{H}), 2.95-2.93(\mathrm{~m}, 2 \mathrm{H}), 2.41-2.34(\mathrm{~m}, 1 \mathrm{H}), 1.68-1.56(\mathrm{~m}$, $2 \mathrm{H}), 1.55-1.48(\mathrm{~m}, 1 \mathrm{H}), 1.33-1.27(\mathrm{~m}, 1 \mathrm{H}) \mathrm{ppm} .{ }^{13} \mathrm{C}$ NMR (100 $\left.\mathrm{MHz}, \mathrm{CD}_{3} \mathrm{CN}\right): \delta=191.3,141.0,133.6,131.5,131.2,130.2$ (2C),
129.9, 128.9 (2C), 120.8, 38.4, 32.1, 21.1, 14.6, 14.1 ppm. HRMS (ESI-TOF) calcd for $\mathrm{C}_{18} \mathrm{H}_{18} \mathrm{~N}\left(\mathrm{M}-\mathrm{BF}_{4}\right)^{+} \mathrm{m} / z=248.1434$, found 248.1455 .

\section{2,4-Diphenyl-3,4-dihydroquinolinium tetrafluoroborate $(3 \mathrm{~h})$}

Orange solid (129 mg, 67\%). Mp 101-102 ${ }^{\circ} \mathrm{C} .{ }^{1} \mathrm{H}$ NMR $(400 \mathrm{MHz}$, $\left.\mathrm{CD}_{3} \mathrm{CN}\right): \delta=12.32$ (brs, $\left.1 \mathrm{H}\right), 8.10-8.08(\mathrm{~m}, 2 \mathrm{H}), 7.90-7.86(\mathrm{~m}$, $1 \mathrm{H}), 7.77$ (dd, $J=8.0,1.6 \mathrm{~Hz}, 1 \mathrm{H}), 7.74-7.70(\mathrm{~m}, 2 \mathrm{H}), 7.56-7.47$ $(\mathrm{m}, 2 \mathrm{H}), 7.42-7.32(\mathrm{~m}, 3 \mathrm{H}), 7.28-7.26(\mathrm{~m}, 2 \mathrm{H}), 7.12(\mathrm{~d}, J=$ $7.6 \mathrm{~Hz}, 1 \mathrm{H}), 4.67$ (t, $J=8.4 \mathrm{~Hz}, 1 \mathrm{H}), 3.97-3.83(\mathrm{~m}, 2 \mathrm{H}) \mathrm{ppm} .{ }^{13} \mathrm{C}$ NMR (100 MHz, $\left.\mathrm{CD}_{3} \mathrm{CN}\right): \delta=179.0,141.1,138.0,133.9,132.7$, 131.7, 130.9 (2C), 130.6 (2C), 130.3, 130.0, 129.1, 129.0, 122.6, 38.8, 36.0 ppm. HRMS (ESI-TOF) calcd for $\mathrm{C}_{21} \mathrm{H}_{18} \mathrm{~N}\left(\mathrm{M}-\mathrm{BF}_{4}\right)^{+} \mathrm{m} / \mathrm{z}$ $=284.1434$, found 284.1439 .

\section{4-(4-Bromophenyl)-2-methyl-3,4-dihydroquinolinium tetrafluoro-borate (3i)}

Orange solid (162 mg, 80\%). Mp 145-147 ${ }^{\circ} \mathrm{C} .{ }^{1} \mathrm{H}$ NMR $(400 \mathrm{MHz}$, $\mathrm{CD}_{3} \mathrm{CN}$ ): $\delta=12.44$ (br, $\left.1 \mathrm{H}, \mathrm{N}-H\right), 7.56-7.42(\mathrm{~m}, 5 \mathrm{H}, \mathrm{Ar}-H), 7.16-$ $7.13(\mathrm{~m}, 2 \mathrm{H}, \mathrm{Ar}-H), 7.02$ (d, $J=7.4 \mathrm{~Hz}, 1 \mathrm{H}, \mathrm{Ar}-H), 4.50,4.52-4.48$ $(\mathrm{t}, J=8.8 \mathrm{~Hz}, 1 \mathrm{H},-\mathrm{CH}), 3.47-3.29\left(\mathrm{~m}, 2 \mathrm{H},-\mathrm{CH}_{2}\right), 2.65(\mathrm{~s}, 3 \mathrm{H}$, $\left.-\mathrm{CH}_{3}\right)$ ppm. ${ }^{13} \mathrm{C}$ NMR $\left(100 \mathrm{MHz}, \mathrm{CD}_{3} \mathrm{CN}\right): \delta=188.7,140.7$, 133.0, 132.7, 132.3, 131.0, 130.3, 129.8(2C), 122.0, 121.4, 38.0, 37.7, 25.3 ppm. HRMS (ESI-TOF) calcd for $\mathrm{C}_{16} \mathrm{H}_{15} \mathrm{~N}^{79} \mathrm{Br}\left(\mathrm{M}-\mathrm{BF}_{4}\right)$ : $m / z=300.0388$, found 300.0379; HRMS (ESI-TOF) calcd for $\mathrm{C}_{16} \mathrm{H}_{15} \mathrm{~N}^{81} \mathrm{Br}\left(\mathrm{M}-\mathrm{BF}_{4}\right): m / z=302.0368$; found: 302.0359 .

\section{4-(4-Chlorophenyl)-2-methyl-3,4-dihydroquinolinium} tetrafluoro-borate $(3 \mathbf{j})$

Yellow solid (111 mg, 62\%). Mp 161-162 ${ }^{\circ} \mathrm{C} .{ }^{1} \mathrm{H}$ NMR $(400 \mathrm{MHz}$, $\left.\mathrm{CD}_{3} \mathrm{CN}\right): \delta=7.51-7.39(\mathrm{~m}, 5 \mathrm{H}, \mathrm{Ar}-H), 7.22-7.19(\mathrm{~m}, 2 \mathrm{H}, \mathrm{Ar}-H)$, $7.03(\mathrm{~d}, J=7.2 \mathrm{~Hz}, 1 \mathrm{H}, \mathrm{Ar}-H), 4.514 .53-4.49(\mathrm{t}, J=8.4 \mathrm{~Hz}, 1 \mathrm{H}$, $-\mathrm{CH}$ ), 3.48-3.29 (m, 2H, $-\mathrm{CH}_{2}$ ), 2.65 (s, 3H, $-\mathrm{CH}_{3}$ ) ppm. ${ }^{13} \mathrm{C} \mathrm{NMR}$ (100 MHz, $\left.\mathrm{CD}_{3} \mathrm{CN}\right): \delta=188.6,140.3,134.0,132.8,132.3,130.72$, 130.4, 130.0, 129.9, 129.4, 121.4, 38.1, 37.7, 25.3 ppm. HRMS (ESI-TOF) calcd for $\mathrm{C}_{16} \mathrm{H}_{15} \mathrm{~N}^{35} \mathrm{Cl}\left(\mathrm{M}-\mathrm{BF}_{4}\right) \mathrm{m} / z=256.0893$, found 256.0897; HRMS (ESI-TOF) calcd for $\mathrm{C}_{16} \mathrm{H}_{15} \mathrm{~N}^{37} \mathrm{Cl}\left(\mathrm{M}-\mathrm{BF}_{4}\right) \mathrm{m} / z=$ 258.0864; found: 258.0964 .

4-(4-(Chloromethyl)phenyl)-2-methyl-3,4-dihydroquinolinium tetrafluoroborate $(3 \mathrm{k})$

Bright yellow solid (141 mg, 76\%). Mp 126-127 ${ }^{\circ} \mathrm{C} .{ }^{1} \mathrm{H}$ NMR (400 $\mathrm{MHz}, \mathrm{CD}_{3} \mathrm{CN}$ ): $\delta=12.29$ (brs, $\left.1 \mathrm{H}\right), 7.53-7.49(\mathrm{~m}, 2 \mathrm{H}), 7.47-7.41$ $(\mathrm{m}, 3 \mathrm{H}), 7.22(\mathrm{~d}, J=8.0 \mathrm{~Hz}, 2 \mathrm{H}), 7.03(\mathrm{~d}, J=7.6 \mathrm{~Hz}, 1 \mathrm{H}), 4.66(\mathrm{~s}$, $2 \mathrm{H}), 4.51(\mathrm{t}, J=8.8 \mathrm{~Hz}, 1 \mathrm{H}), 3.48-3.31(\mathrm{~m}, 2 \mathrm{H}), 2.65(\mathrm{~s}, 3 \mathrm{H}) \mathrm{ppm}$. ${ }^{13} \mathrm{C}$ NMR $\left(100 \mathrm{MHz}, \mathrm{CD}_{3} \mathrm{CN}\right): \delta=189.0,142.1,138.9,133.1$, 132.7, 131.0, 130.8, 130.3, 130.2, 129.7, 121.8, 46.9, 38.5, 38.4, $25.7 \mathrm{ppm}$. HRMS (ESI-TOF) calcd for $\mathrm{C}_{17} \mathrm{H}_{17}{ }^{35} \mathrm{ClN}(\mathrm{M}-\mathrm{BF} 4)^{+} \mathrm{m} / \mathrm{z}$ $=270.1044$, found 270.1051; HRMS (ESI-TOF) calcd for $\mathrm{C}_{17} \mathrm{H}_{17}{ }^{37} \mathrm{ClN}(\mathrm{M}-\mathrm{BF} 4)^{+} \mathrm{m} / z=272.1044$, found 272.1115 .

4-(2,5-Dimethylphenyl)-2-isopropyl-3,4-dihydroquinolinium tetrafluoroborate (31)

Orange solid (74 mg, 39\%). Mp 158-159 ${ }^{\circ} \mathrm{C} .{ }^{1} \mathrm{H}$ NMR $(400 \mathrm{MHz}$, $\left.\mathrm{CD}_{3} \mathrm{CN}\right): \delta=12.04(\mathrm{br}, 1 \mathrm{H}, \mathrm{N}-H), 7.64-7.62(\mathrm{~d}, J=7.6 \mathrm{~Hz}, 1 \mathrm{H}$, 
$\mathrm{Ar}-H)$, 7.53-7.44 (m, 2H, Ar-H), 7.19-7.17 (d, $=8.0 \mathrm{~Hz}, 1 \mathrm{H}, \mathrm{Ar}-$ $H)$, 7.05-7.00 (m, 2H, Ar-H), $6.65(\mathrm{~s}, 1 \mathrm{H}, \mathrm{Ar}-H), 4.74-4.70(\mathrm{t}, J=$ $8.4 \mathrm{~Hz}, 1 \mathrm{H},-\mathrm{CH}), 3.41-3.24\left(\mathrm{~m}, 2 \mathrm{H},-\mathrm{CH}_{2}\right), 3.21-3.11$ (septet, $J=$ $\left.6.8 \mathrm{~Hz}, 1 \mathrm{H},-\mathrm{C} H\left(\mathrm{CH}_{3}\right)\left(\mathrm{CH}_{3}\right)\right), 2.37\left(\mathrm{~s}, 3 \mathrm{H},-\mathrm{CH}_{3}\right), 2.19(\mathrm{~s}, 3 \mathrm{H}$, $\left.-\mathrm{CH}_{3}\right), 1.29-1.27\left(\mathrm{~d}, J=6.8 \mathrm{~Hz}, 3 \mathrm{H},-\mathrm{CH}_{3}\right), 1.18-1.16(\mathrm{~d}, J=$ $\left.6.8 \mathrm{~Hz}, 3 \mathrm{H},-\mathrm{CH}_{3}\right) \mathrm{ppm} .{ }^{13} \mathrm{C} \mathrm{NMR}\left(100 \mathrm{MHz}, \mathrm{CD}_{3} \mathrm{CN}\right): \delta=194.8$, 138.6, 137.3, 134.1, 133.4, 132.5, 131.9, 131.6, 129.7 (2C), 129.3, 128.5, 122.0, 38.2, 34.3, 33.7, 20.9, 19.1, 18.5, 18.0 ppm. HRMS (ESI-TOF) calcd for $\mathrm{C}_{20} \mathrm{H}_{24}\left(\mathrm{M}-\mathrm{BF}_{4}\right)$ : $=278.1909$; found: 278.1906.

\section{2,4-Dimethyl-4-phenyl-3,4-dihydroquinolinium tetrafluoroborate $(3 \mathrm{~m})$}

White solid (60 mg, 49\%). Mp 171-172 ${ }^{\circ} \mathrm{C} .{ }^{1} \mathrm{H}$ NMR $(400 \mathrm{MHz}$, $\left.\mathrm{CD}_{3} \mathrm{CN}\right): \delta=7.55-7.51(\mathrm{~m}, 3 \mathrm{H}, \mathrm{Ar}-H), 7.37-7.29(\mathrm{~m}, 4 \mathrm{H}, \mathrm{Ar}-H)$, 7.21-7.19 (m, 2H, Ar-H), 3.71-3.66 (d, $J=18.8 \mathrm{~Hz}, 1 \mathrm{H}$, $\left.-\mathrm{CH}_{\mathrm{a}}\left(H_{b}\right)\right), 3.23-3.19\left(\mathrm{~d}, J=18.8 \mathrm{~Hz}, 1 \mathrm{H},-\mathrm{C}\left(H_{\mathrm{a}}\right) H_{b}\right), 2.63(\mathrm{~s}, 3 \mathrm{H}$, $\left.-\mathrm{CH}_{3}\right), 1.72\left(\mathrm{~s}, 3 \mathrm{H},-\mathrm{CH}_{3}\right) \mathrm{ppm} .{ }^{13} \mathrm{C} \mathrm{NMR}\left(100 \mathrm{MHz}, \mathrm{CD}_{3} \mathrm{CN}\right): \delta=$ $188.3,144.8,135.8,132.5,132.4$, 129.7, 129.6, 128.6, 128.3, 127.3, 121.7, 44.8, 40.0, 27.3, 25.4 ppm. HRMS (ESI-TOF) calcd for $\mathrm{C}_{17} \mathrm{H}_{18} \mathrm{~N}\left(\mathrm{M}-\mathrm{BF}_{4}\right)$ : = 236.1439; found 236.1442.

\section{6-Methyl-6a,7,8,9,10,10a-hexahydro-7,10- methanophenanthridinum tetrafluoroborate (3n)}

Pale yellow solid (95 mg, 62\%). Mp 191-192 ${ }^{\circ} \mathrm{C} .{ }^{1} \mathrm{H}$ NMR (400 $\left.\mathrm{MHz}, \mathrm{CD}_{3} \mathrm{CN}\right): \delta=12.0(\mathrm{brs}, 1 \mathrm{H}), 7.45(\mathrm{t}, J=7.6 \mathrm{~Hz}, 1 \mathrm{H}), 7.40(\mathrm{~d}$, $J=7.2 \mathrm{~Hz}, 1 \mathrm{H}), 7.34(\mathrm{t}, J=7.2 \mathrm{~Hz}, 1 \mathrm{H}), 7.29(\mathrm{~d}, J=7.6 \mathrm{~Hz}, 1 \mathrm{H})$, $3.28(\mathrm{~d}, J=10.4 \mathrm{~Hz}, 1 \mathrm{H}), 3.20(\mathrm{~d}, J=10.4 \mathrm{~Hz}, 1 \mathrm{H}), 2.85(\mathrm{~s}, 1 \mathrm{H})$, $2.59(\mathrm{~s}, 3 \mathrm{H}), 2.47(\mathrm{~s}, 1 \mathrm{H}), 1.79-1.62(\mathrm{~m}, 4 \mathrm{H}), 1.35-1.28(\mathrm{~m}$, $2 \mathrm{H}) \mathrm{ppm} .{ }^{13} \mathrm{C}$ NMR $\left(100 \mathrm{MHz}, \mathrm{CD}_{3} \mathrm{CN}\right): \delta=186.5,132.5,131.3$, 131.0, 129.4, 129.1, 121.4, 50.1, 49.5, 47.1, 42.9, 36.3, 31.2, 29.3, 24.3 ppm. HRMS (ESI-TOF) calcd for $\mathrm{C}_{15} \mathrm{H}_{18} \mathrm{~N}\left(\mathrm{M}-\mathrm{BF}_{4}\right)^{+} \mathrm{m} / z=$ 212.1434, found 212.1445 .

\section{2,4-Diphenylquinazoline $(4)^{18 c}$}

White solid (26 mg, 18\%). Mp 110-111 ${ }^{\circ} \mathrm{C} .{ }^{1} \mathrm{H}$ NMR $(400 \mathrm{MHz}$, $\left.\mathrm{CDCl}_{3}\right): \delta=8.70(\mathrm{~d}, J=8.0 \mathrm{~Hz}, 2 \mathrm{H}), 8.15(\mathrm{~d}, J=8.4 \mathrm{~Hz}, 1 \mathrm{H}), 8.12$ $(\mathrm{d}, J=8.4 \mathrm{~Hz}, 1 \mathrm{H}), 7.89-7.85(\mathrm{~m}, 3 \mathrm{H}), 7.59-7.57$ (m, 3H), 7.55$7.47(\mathrm{~m}, 4 \mathrm{H}) \mathrm{ppm} .{ }^{13} \mathrm{C}$ NMR $\left(100 \mathrm{MHz}, \mathrm{CDCl}_{3}\right) \delta$ 168.3, 160.2, 151.9, 138.1, 137.6, 133.5, 130.5, 130.1, 129.9, 129.1, 128.6, 128.5 (3C), 126.9, 121.6 ppm. HRMS (ESI-TOF) calcd for $\mathrm{C}_{20} \mathrm{H}_{15} \mathrm{~N}_{2}(\mathrm{M}+$ $\mathrm{H})^{+} m / z=283.1235$, found 283.1231. These data are in agreement with the reported data. ${ }^{18 c}$

\section{2-Methyl-4-phenylquinoline (5)}

Pale yellow solid (33 mg, 77\%). ${ }^{1} \mathrm{H}$ NMR (400 MHz, $\left.\mathrm{CDCl}_{3}\right): \delta=$ 8.07 (d, $J=8.8 \mathrm{~Hz}, 1 \mathrm{H}), 7.82(\mathrm{~d}, J=8.4 \mathrm{~Hz}, 1 \mathrm{H}), 7.66-7.63(\mathrm{~m}$, 1H), 7.49-7.43 (m 5H), 7.38 (t, J= 7.2 Hz, 1H), 7.19 (s, 1H), 2.75 $(\mathrm{s}, 3 \mathrm{H}) .{ }^{13} \mathrm{C} \mathrm{NMR}\left(100 \mathrm{MHz}, \mathrm{CDCl}_{3}\right): \delta=158.4,148.4,148.3$, 138.0, 129.4, 129.2, 128.9, 128.4, 128.2, 125.6, 125.5, 125.0, 122.1, 25.2; HRMS (ESI) calcd for $\mathrm{C}_{16} \mathrm{H}_{14} \mathrm{~N}(\mathrm{M}+\mathrm{H})^{+} \mathrm{m} / z=$ 220.1126 found 220.1127 . These data are in agreement with the reported data. ${ }^{18 b}$
General procedure for the oxidation of 3,4-dihydroquinoline with oxygen

2-Methyl-4-phenyl-3,4-dihydroquinolinium tetrafluoroborate 3a $(0.3 \mathrm{mmol})$ was extracted with DCM $(25 \mathrm{~mL})$, washed with a saturated aqueous solution of $\mathrm{NaHCO}_{3}(5 \mathrm{~mL})$. The organic layer was dried over $\mathrm{MgSO}_{4}$ and filtered. To this was added $\mathrm{Na}_{2} \mathrm{CO}_{3}$ (10 equiv) and this mixture was stirred at room temperature for 3 days. Next, the resulting mixture was filtered through a plug of Celite and concentrated in vacuo. The residue was purified by flash column chromatography (silica gel, eluent, $30 \%$ EtOAc in hexane) to give the desired product.

\section{General procedure for one-pot preparation of 3- hydroxyquinoline}

In a dry $10 \mathrm{~mL}$ glass sealed tube, phenyldiazonium salt $(100 \mathrm{mg}$, $0.52 \mathrm{mmol}, 1.0$ eq.) and styrene (108 mg, $1.04 \mathrm{mmol}, 2.0$ eq.) were suspended in $2 \mathrm{~mL}$ of anhydrous nitrile. The tube was sealed with a Teflon screw cap and heated in an oil bath $\left(80^{\circ} \mathrm{C}\right)$ for $2 \mathrm{~h}$. After cooling to room temperature, reaction mixture was diluted with DCM $(20 \mathrm{~mL})$ and washed with saturated aq. $\mathrm{NaHCO}_{3}$ solution $(10 \mathrm{~mL})$. Combined organic layer was stirred over anhydrous $\mathrm{Na}_{2} \mathrm{CO}_{3}(5 \mathrm{~g})$ for 3 days. Solvents removed and purified by column chromatography (eluent: $20 \%$ to $40 \%$ EtOAc in hexane) to obtain the corresponding substituted 3hydroxyquinolines.

\section{2-Methyl-4-phenylquinolin-3-ol (6a) $)^{18 b}$}

Off-white solid (44 mg, 36\%); mp 238-239 ${ }^{\circ} \mathrm{C}:{ }^{1} \mathrm{H}$ NMR (400 MHz, DMSO- $\left.d_{6}\right) \delta 8.86$ (brs, $\left.1 \mathrm{H}\right), 7.87(\mathrm{~d}, J=8.4 \mathrm{~Hz}, 1 \mathrm{H}), 7.54(\mathrm{t}, J$ $=7.2 \mathrm{~Hz}, 2 \mathrm{H}), 7.47(\mathrm{t}, J=7.2 \mathrm{~Hz}, 2 \mathrm{H}), 7.34-7.31(\mathrm{~m}, 3 \mathrm{H}), 7.23(\mathrm{~d}$, $J=8.0 \mathrm{~Hz}, 1 \mathrm{H}), 2.62(\mathrm{~s}, 3 \mathrm{H}) ;{ }^{13} \mathrm{C}$ NMR (100 MHz, DMSO- $\left.d_{6}\right)$ $\delta 152.5,145.4,142.1,133.7,130.3,128.7,128.4,128.2,127.8$, 127.5, 125.8, 125.7, 124.1, 21.2. HRMS (ESI-TOF) calcd for $\mathrm{C}_{16} \mathrm{H}_{14} \mathrm{NO}(\mathrm{M}+\mathrm{H})^{+} \mathrm{m} / z=236.1075$, found 236.1073. These data are in agreement with the reported data.

\section{4-(4-(Chloromethyl)phenyl)-2-methylquinolin-3-ol (6b) ${ }^{15}$}

Pale yellow solid (59 mg, 69\%). ${ }^{1} \mathrm{H}$ NMR (400 MHz, DMSO- $d_{6}$ ): $\delta=8.98(\mathrm{br}, 1 \mathrm{H}, \mathrm{O}-H), 7.88(\mathrm{~d}, J=8.4 \mathrm{~Hz}, 1 \mathrm{H}, \mathrm{Ar}-H), 7.60(\mathrm{~d}, J=$ $8.0 \mathrm{~Hz}, 2 \mathrm{H}, \mathrm{Ar}-H)$, 7.50-7.46 (m, $1 \mathrm{H}, \mathrm{Ar}-H), 7.37-7.33(\mathrm{~m}, 3 \mathrm{H}$, $\mathrm{Ar}-H), 7.23(\mathrm{~d}, J=8.4 \mathrm{~Hz}, 1 \mathrm{H}, \mathrm{Ar}-H), 4.88$ (s, 2H, $\left.-\mathrm{CH}_{2} \mathrm{Cl}\right), 2.63$ (s, $\left.3 \mathrm{H},-\mathrm{CH}_{3}\right) \mathrm{ppm} .{ }^{13} \mathrm{C} \mathrm{NMR}\left(100 \mathrm{MHz}, \mathrm{DMSO}-d_{6}\right): \delta=152.6,145.5$, 142.1, 137.1, 133.9, 130.7, 129.0, 128.3, 128.2, 127.4, 125.8(2C), 124.0, 46.0, 21.3 ppm. HRMS (ESI-TOF) calcd for $\mathrm{C}_{17} \mathrm{H}_{15}{ }^{35} \mathrm{ClNO}$ $\left(\mathrm{M}+\mathrm{H}^{+}\right) m / z=284.0842$; found: 284.0840; HRMS (ESI-TOF) calcd for $\mathrm{C}_{17} \mathrm{H}_{15}{ }^{37} \mathrm{ClNO}\left(\mathrm{M}+\mathrm{H}^{+}\right) \mathrm{m} / z=286.0813$; found: 286.0807. These data are in agreement with the reported data.

\section{4-(4-Chlorophenyl)-2-methylquinolin-3-ol (6c)}

Pale yellow solid (41 mg, 50\%). Mp 272-274 ${ }^{\circ} \mathrm{C} .{ }^{1} \mathrm{H}$ NMR (400 MHz, DMSO- $\left.d_{6}\right): \delta=7.87(\mathrm{~d}, J=8.4 \mathrm{~Hz}, 1 \mathrm{H}, \mathrm{Ar}-H), 7.59(\mathrm{~d}, J=$ $8.4 \mathrm{~Hz}, 2 \mathrm{H}, \mathrm{Ar}-H)$, 7.50-7.46 (m, $1 \mathrm{H}, \mathrm{Ar}-H), 7.38-7.33(\mathrm{~m}, 3 \mathrm{H}$, $\mathrm{Ar}-H), 7.23(\mathrm{~d}, J=8.0 \mathrm{~Hz}, 1 \mathrm{H}, \mathrm{Ar}-H), 2.61\left(\mathrm{~s}, 3 \mathrm{H},-\mathrm{CH}_{3}\right) \mathrm{ppm} .{ }^{13} \mathrm{C}$ NMR (125 MHz, DMSO- $\left.d_{6}\right): \delta=152.6,145.7,142.0,132.7,132.4$, 128.6, 128.3, 127.3, 127.2, 126.0, 125.9(2C), 123.9, 21.2 ppm. 
HRMS (ESI-TOF) calcd for $\mathrm{C}_{16} \mathrm{H}_{13}{ }^{35} \mathrm{ClNO}\left(\mathrm{M}+\mathrm{H}^{+}\right) \mathrm{m} / \mathrm{z}=$ 270.0680; found: 270.0695; HRMS (ESI-TOF) calcd for $\mathrm{C}_{16} \mathrm{H}_{13}{ }^{37} \mathrm{ClNO}\left(\mathrm{M}+\mathrm{H}^{+}\right) \mathrm{m} / \mathrm{z}=272.0651$; found: 272.0692 .

\section{2,6-Dimethyl-4-phenylquinolin-3-ol (6d)}

Pale yellow solid (56 mg, 47\%). Mp 205-206 ${ }^{\circ} \mathrm{C} .{ }^{1} \mathrm{H}$ NMR (400 $\left.\mathrm{MHz}, \mathrm{CDCl}_{3}\right): \delta=7.92(\mathrm{~d}, J=8.4 \mathrm{~Hz}, 1 \mathrm{H}), 7.57-7.47(\mathrm{~m}, 3 \mathrm{H})$, 7.37-7.32 (m, 3H), $7.06(\mathrm{~s}, 1 \mathrm{H}), 4.32(\mathrm{brs}, 1 \mathrm{H}), 2.71(\mathrm{~s}, 3 \mathrm{H}), 2.35$ (s, 3H) ppm. ${ }^{13} \mathrm{C}$ NMR $\left(100 \mathrm{MHz}, \mathrm{CDCl}_{3}\right): \delta=150.3,144.8,141.0$, 136.0, 135.8, 132.4, 130.2, 129.4, 128.8, 128.5, 127.9, 127.2, 123.3, 21.6, $20.4 \mathrm{ppm}$. HRMS (ESI-TOF) calcd for $\mathrm{C}_{17} \mathrm{H}_{16} \mathrm{NO}(\mathrm{M}+$ $\mathrm{H})^{+} m / z=250.1232$, found 250.1239 .

\section{6-(tert-Butyl)-2-methyl-4-phenylquinolin-3-ol (6e)}

Pale yellow solid (52 mg, 44\%). Mp 243-244 ${ }^{\circ} \mathrm{C} .{ }^{1} \mathrm{H}$ NMR (400 $\left.\mathrm{MHz}, \mathrm{CDCl}_{3}\right): \delta=7.90(\mathrm{~d}, J=9.2 \mathrm{~Hz}, 1 \mathrm{H}), 7.59-7.52(\mathrm{~m}, 2 \mathrm{H})$, $7.47(\mathrm{t}, J=7.6 \mathrm{~Hz}, 2 \mathrm{H}), 7.38(\mathrm{~d}, J=7.6 \mathrm{~Hz}, 2 \mathrm{H}), 7.25-7.24(\mathrm{~m}$, $1 \mathrm{H}), 2.68(\mathrm{~s}, 3 \mathrm{H}), 1.24(\mathrm{~s}, 9 \mathrm{H}) \mathrm{ppm} .{ }^{13} \mathrm{C} \mathrm{NMR}\left(100 \mathrm{MHz}, \mathrm{CDCl}_{3}\right)$ : $\delta=150.4,148.7,144.6,141.2,132.3,130.2,129.5,128.9,127.9$, $127.5,126.7,125.1,119.4,34.8,31.0,20.5$ ppm. HRMS (ESI-TOF) calcd for $\mathrm{C}_{20} \mathrm{H}_{20} \mathrm{NO}(\mathrm{M}+\mathrm{H})^{+} \mathrm{m} / \mathrm{z}=292.1701$, found 292.1702.

\section{4-Phenyl-2-propylquinolin-3-ol (6f)}

Pale yellow solid (43 mg, 32\%). Mp 177-178 ${ }^{\circ} \mathrm{C} .{ }^{1} \mathrm{H}$ NMR (400 $\left.\mathrm{MHz}, \mathrm{CDCl}_{3}\right): \delta=8.00(\mathrm{~d}, J=8.4 \mathrm{~Hz}, 1 \mathrm{H}), 7.57-7.54(\mathrm{~m}, 2 \mathrm{H})$, 7.50-7.46 (m, 2H), 7.38 (d, $J=7.2 \mathrm{~Hz}, 2 \mathrm{H}), 7.32-7.31(\mathrm{~m}, 2 \mathrm{H})$, 5.50 (brs, $1 \mathrm{H}$ ), 3.02 (t, $J=8.0 \mathrm{~Hz}, 2 \mathrm{H}), 1.85$ (sextet, $J=7.6 \mathrm{~Hz}$, $2 \mathrm{H}), 1.05(\mathrm{t}, J=7.6 \mathrm{~Hz}, 3 \mathrm{H}) \mathrm{ppm} .{ }^{13} \mathrm{C}$ NMR $\left(100 \mathrm{MHz}, \mathrm{CDCl}_{3}\right)$ $\delta$ 154.7, 144.3, 142.9, 132.2, 130.3, 129.6, 129.0, 128.7, 127.5, 127.1, 126.2, 126.0, 124.3, 36.1, 21.7, 14.2 ppm. HRMS (ESI-TOF) calcd for $\mathrm{C}_{18} \mathrm{H}_{18} \mathrm{NO}(\mathrm{M}+\mathrm{H})^{+} \mathrm{m} / \mathrm{z}=264.1388$, found 264.1390.

\section{2-Isopropyl-4-phenylquinolin-3-ol (6g)}

Pale yellow solid (53 mg, 39\%). Mp 98-99 ${ }^{\circ} \mathrm{C} .{ }^{1} \mathrm{H}$ NMR (400 MHz, $\left.\mathrm{CDCl}_{3}\right): \delta=8.05(\mathrm{~d}, J=8.4 \mathrm{~Hz}, 1 \mathrm{H}), 7.61-7.57(\mathrm{~m}, 2 \mathrm{H}), 7.54-7.47$ $(\mathrm{m}, 2 \mathrm{H}), 7.40(\mathrm{~d}, J=6.8 \mathrm{~Hz}, 2 \mathrm{H}), 7.32-7.31(\mathrm{~m}, 2 \mathrm{H}), 5.25$ (brs, $1 \mathrm{H}), 3.63$ (sept, $J=6.8 \mathrm{~Hz}, 1 \mathrm{H}), 1.43(\mathrm{~d}, J=6.8 \mathrm{~Hz}, 6 \mathrm{H}) \mathrm{ppm} .{ }^{13} \mathrm{C}$ NMR $\left(100 \mathrm{MHz}, \mathrm{CDCl}_{3}\right): \delta=158.5,143.6,142.9,132.2,130.4$, $129.7,129.1,129.0,127.2,126.9,126.0,125.9,124.2,31.0$, $20.8 \mathrm{ppm}$. HRMS (ESI-TOF) calcd for $\mathrm{C}_{18} \mathrm{H}_{18} \mathrm{NO}(\mathrm{M}+\mathrm{H})^{+} \mathrm{m} / \mathrm{z}=$ 264.1388, found 264.1394.

\section{2-Cyclopropyl-4-phenylquinolin-3-ol (6h)}

Off-white solid (57 mg, 42\%). Mp 127-128 ${ }^{\circ} \mathrm{C} .{ }^{1} \mathrm{H}$ NMR $(400$ $\left.\mathrm{MHz}, \mathrm{CDCl}_{3}\right): \delta=7.97(\mathrm{~d}, J=8.4 \mathrm{~Hz}, 1 \mathrm{H}), 7.58(\mathrm{t}, J=7.6 \mathrm{~Hz}, 2 \mathrm{H})$, 7.54-7.44 (m, 2H), 7.41-7.39 (m, 2H), 7.31-7.26 (m, 2H), 5.35 (brs, 1H), $2.63(\mathrm{~m}, 1 \mathrm{H}), 1.36-1.35(\mathrm{~m}, 2 \mathrm{H}), 1.10-1.06(\mathrm{~m}, 2 \mathrm{H}) .{ }^{13} \mathrm{C}$ NMR $\left(100 \mathrm{MHz}, \mathrm{CDCl}_{3}\right): \delta=154.5,144.4,132.2,130.3,129.9$ (2C), 129.6, 129.0, 128.4, 126.6, 126.3, 125.6, 124.3, 11.9, 9.5. HRMS (ESI-TOF) calcd for $\mathrm{C}_{18} \mathrm{H}_{16} \mathrm{NO}(\mathrm{M}+\mathrm{H})^{+} \mathrm{m} / \mathrm{z}=262.1232$, found 262.1232 .

\section{4-Hexyl-2-methylquinolin-3-ol (6i)}

Off-white solid (70 mg, 56\%). Mp 161-162 ${ }^{\circ} \mathrm{C} .{ }^{1} \mathrm{H}$ NMR (400 $\left.\mathrm{MHz}, \mathrm{CDCl}_{3}\right): \delta=8.00(\mathrm{~d}, J=8.0 \mathrm{~Hz}, 1 \mathrm{H}), 7.83(\mathrm{dd}, J=8.0$,
$1.2 \mathrm{~Hz}, 1 \mathrm{H}), 7.51-7.44(\mathrm{~m}, 2 \mathrm{H}), 3.02(\mathrm{t}, J=8.0 \mathrm{~Hz}, 2 \mathrm{H}), 2.67(\mathrm{~s}$, $3 \mathrm{H}), 1.66-1.58(\mathrm{~m}, 2 \mathrm{H}), 1.43-1.36(\mathrm{~m}, 2 \mathrm{H}), 1.28-1.26(\mathrm{~m}, 2 \mathrm{H})$, 1.25-1.23 (m, 2H), $0.84(\mathrm{t}, J=6.8 \mathrm{~Hz}, 3 \mathrm{H}) \mathrm{ppm} .{ }^{13} \mathrm{C}$ NMR $(100$ $\left.\mathrm{MHz}, \mathrm{CDCl}_{3}\right): \delta=150.2,146.1,141.5,127.6$ (2C), 126.9, 126.3, 122.9 (2C), 31.6, 29.6, 29.3, 25.2, 22.5, 20.0, 14.0 ppm. HRMS (ESI-TOF) calcd for $\mathrm{C}_{16} \mathrm{H}_{22} \mathrm{NO}(\mathrm{M}+\mathrm{H})^{+} \mathrm{m} / z=244.1701$, found 244.1696.

\section{2,4-Diphenylquinolin-3-ol (6j) $)^{20}$}

Off-white solid (68 mg, 46\%). Mp 123-124 ${ }^{\circ} \mathrm{C} .{ }^{1} \mathrm{H}$ NMR (400 $\left.\mathrm{MHz}, \mathrm{CDCl}_{3}\right): \delta=8.15(\mathrm{~d}, J=8.4 \mathrm{~Hz}, 1 \mathrm{H}), 8.01-7.93(\mathrm{~m}, 2 \mathrm{H})$, 7.62-7.58 (m, 2H), 7.58-7.53 (m, 2H), 7.52-7.48 (m, 2H), 7.48$7.42(\mathrm{~m}, 3 \mathrm{H}), 7.42-7.37$ (m, 2H), 5.45 (brs, $1 \mathrm{H}) .{ }^{13} \mathrm{C}$ NMR $(100$ $\left.\mathrm{MHz}, \mathrm{CDCl}_{3}\right): \delta=150.0,143.9,143.3,137.2,132.2,130.3$ (2C), 129.5 (2C), 129.4 (2C), 129.1, 128.4 (2C), 127.6, 126.8, 124.5. HRMS (ESI-TOF) calcd for $\mathrm{C}_{21} \mathrm{H}_{16} \mathrm{NO}(\mathrm{M}+\mathrm{H})^{+} \mathrm{m} / z=298.1232$, found 298.1242. These data are in agreement with the reported data.

\section{2,4-Dimethyl-4-phenylquinolin-3(4H)-one (7)}

Yellow oil (52 mg, 70\%). ${ }^{1} \mathrm{H}$ NMR $\left(400 \mathrm{MHz}, \mathrm{CDCl}_{3}\right): \delta=7.60(\mathrm{~d}$, $J=7.2 \mathrm{~Hz}, 1 \mathrm{H}, \mathrm{Ar}-H), 7.46-7.42(\mathrm{~m}, 1 \mathrm{H}, \mathrm{Ar}-H), 7.37-7.33(\mathrm{~m}, 1 \mathrm{H}$, $\mathrm{Ar}-H), 7.29-7.23(\mathrm{~m}, 4 \mathrm{H}, \mathrm{Ar}-H), 7.03-7.01$ (m, $2 \mathrm{H}, \mathrm{Ar}-H), 2.28(\mathrm{~s}$, $\left.3 \mathrm{H},-\mathrm{CH}_{3}\right), 1.89\left(\mathrm{~s}, 3 \mathrm{H},-\mathrm{CH}_{3}\right)$ ppm. ${ }^{13} \mathrm{C} \mathrm{NMR}\left(100 \mathrm{MHz}, \mathrm{CDCl}_{3}\right)$ : $\delta=196.4,160.7,140.7,140.6,136.8,129.4,128.8,128.7,128.4$, $127.9,127.6,127.2,53.6,23.3,20.0$ ppm. HRMS (ESI-TOF) calcd for $\mathrm{C}_{17} \mathrm{H}_{16} \mathrm{NO}\left(\mathrm{M}+\mathrm{H}^{+}\right) \mathrm{m} / z=250.1232$; found: 250.1243.

\section{6-Methylphenanthridine ( 8$)^{18 b}$}

Pale yellow oil (59 mg, 82\%). ${ }^{1} \mathrm{H}$ NMR $\left(400 \mathrm{MHz}, \mathrm{CDCl}_{3}\right): \delta=$ 8.59 (d, $J=8.0 \mathrm{~Hz}, 1 \mathrm{H}), 8.50(\mathrm{~d}, J=8.4 \mathrm{~Hz}, 1 \mathrm{H}), 8.18(\mathrm{~d}, J=$ $8.4 \mathrm{~Hz}, 1 \mathrm{H}), 8.09(\mathrm{~d}, J=8.0 \mathrm{~Hz}, 1 \mathrm{H}), 7.81(\mathrm{t}, J=8.0 \mathrm{~Hz}, 1 \mathrm{H}), 7.70-$ $7.64(\mathrm{~m}, 2 \mathrm{H}), 7.59(\mathrm{t}, J=7.6 \mathrm{~Hz}, 1 \mathrm{H}), 3.02(\mathrm{~s}, 3 \mathrm{H}) \mathrm{ppm} .{ }^{13} \mathrm{C} \mathrm{NMR}$ $\left(100 \mathrm{MHz}, \mathrm{CDCl}_{3}\right): \delta=158.8,143.6,132.5,130.4,129.2,128.6$, $127.2,126.5,126.3,125.8,123.7,122.2,121.9,23.3 \mathrm{ppm}$. HRMS(ESI) calcd for $\mathrm{C}_{14} \mathrm{H}_{12} \mathrm{~N}(\mathrm{M}+1)^{+} \mathrm{m} / z=194.0970$, found 194.0976. These data are in agreement with the reported data.

\section{2-Phenylbenzo $[d]$ thiazole $(9)^{21 b}$}

Off-white solid (45 mg, 52\%). Mp 111-112 ${ }^{\circ} \mathrm{C} .{ }^{1} \mathrm{H}$ NMR (400 $\left.\mathrm{MHz}, \mathrm{CDCl}_{3}\right): \delta=8.09-8.05(\mathrm{~m}, 3 \mathrm{H}), 7.88(\mathrm{dd}, J=8.0,0.4 \mathrm{~Hz}$, $1 \mathrm{H}), 7.49-7.45(\mathrm{~m}, 4 \mathrm{H}), 7.36(\mathrm{t}, J=7.2 \mathrm{~Hz}, 1 \mathrm{H}) \mathrm{ppm} .{ }^{13} \mathrm{C} \mathrm{NMR}$ (100 MHz, CDCl3) $\delta 168.0,154.1,135.0,133.5,130.9,128.9$, 127.5, 126.2, 125.1, 123.0, 121.5 ppm. HRMS(ESI) calcd for $\mathrm{C}_{13} \mathrm{H}_{10} \mathrm{NS}(\mathrm{M}+1)^{+} \mathrm{m} / z=212.0534$, found 212.0539. These data are in agreement with the reported data.

\section{General procedure for preparing 2-substituted quinolines from $o$-vinylaryldiazonium tetrafluoroborate}

In a dry $10 \mathrm{~mL}$ glass sealed tube, $o$-vinylaryldiazonium salt ( 0.46 $\mathrm{mmol}$ ) is suspended in $2 \mathrm{~mL}$ of anhydrous nitrile. The tube was sealed with a Teflon screw cap and heated in an oil bath $\left(60^{\circ} \mathrm{C}\right)$ for $12 \mathrm{~h}$. After cooling to room temperature, reaction mixture was diluted with DCM $(20 \mathrm{~mL})$ and washed with saturated aq. $\mathrm{NaHCO}_{3}$ solution $(10 \mathrm{~mL})$. Organic layer was dried over 
anhydrous $\mathrm{Na}_{2} \mathrm{SO}_{4}$. Solvents removed and purified by column chromatography (eluent: $20 \%$ EtOAc : hexane) to obtain the title compound.

\section{2-Methylquinoline (12a) ${ }^{21 c}$}

Brown oil (55 mg, 74\%). ${ }^{1} \mathrm{H}$ NMR $\left(400 \mathrm{MHz}, \mathrm{CDCl}_{3}\right): \delta=7.97$ (d, $J=8.4 \mathrm{~Hz}, 1 \mathrm{H}), 7.92(\mathrm{t}, J=8.0 \mathrm{~Hz}, 1 \mathrm{H}), 7.60(\mathrm{~d}, J=8.0 \mathrm{~Hz}, 1 \mathrm{H})$, 7.61-7.57 (m, 1H), 7.40-7.36 (m, 1H), 7.18-7.15 (m, 1H), 2.66 (s, $3 \mathrm{H}) \mathrm{ppm} .{ }^{13} \mathrm{C}$ NMR $\left(100 \mathrm{MHz}, \mathrm{CDCl}_{3}\right) ; \delta=158.7,147.6,135.9$, 129.2, 128.3, 127.2, 126.2, 125.4, 121.7, 25.1 ppm. HRMS (ESITOF) calcd for $\mathrm{C}_{10} \mathrm{H}_{10} \mathrm{~N}(\mathrm{M}+\mathrm{H})^{+} \mathrm{m} / z=144.0813$, found 144.0818. These data are in agreement with the reported data.

\section{2-Propylquinoline (12b) $)^{21 d}$}

Pale yellow oil (61 mg, 69\%). ${ }^{1} \mathrm{H}$ NMR (400 MHz, $\left.\mathrm{CDCl}_{3}\right): \delta=$ $8.04(\mathrm{dd}, J=8.4,3.2 \mathrm{~Hz}, 2 \mathrm{H}), 7.75(\mathrm{~d}, J=8.4 \mathrm{~Hz}, 1 \mathrm{H}), 7.68-7.63$ $(\mathrm{m}, 1 \mathrm{H}), 7.45(\mathrm{t}, J=8.0 \mathrm{~Hz}, 1 \mathrm{H}), 7.27(\mathrm{~d}, J=8.4 \mathrm{~Hz}, 1 \mathrm{H}), 2.94(\mathrm{t}, J$ $=7.6 \mathrm{~Hz}, 2 \mathrm{H}), 1.82(\mathrm{sext}, J=7.6 \mathrm{~Hz}, 2 \mathrm{H}), 1.00(\mathrm{t}, J=7.6 \mathrm{~Hz}$, $3 \mathrm{H}) \mathrm{ppm} .{ }^{13} \mathrm{C}$ NMR $\left(100 \mathrm{MHz}, \mathrm{CDCl}_{3}\right): \delta=162.8,147.7,136.2$, 129.3, 128.6, 127.4, 126.7, 125.6, 121.3, 41.1, 23.2, 13.9 ppm. HRMS (ESI-TOF) calcd for $\mathrm{C}_{12} \mathrm{H}_{14} \mathrm{~N}(\mathrm{M}+\mathrm{H})^{+} \mathrm{m} / z=172.1126$, found 172.1126. These data are in agreement with the reported data.

\section{2-Isopropylquinoline (12c) $)^{21 e}$}

Pale yellow oil (56 mg, 63\%). ${ }^{1} \mathrm{H}$ NMR $\left(400 \mathrm{MHz}, \mathrm{CDCl}_{3}\right): \delta=$ $8.05(\mathrm{~d}, J=8.4 \mathrm{~Hz}, 1 \mathrm{H}), 8.00(\mathrm{~d}, J=8.4 \mathrm{~Hz}, 1 \mathrm{H}), 7.70(\mathrm{~d}, J=$ $8.0 \mathrm{~Hz}, 1 \mathrm{H}), 7.65-7.61(\mathrm{~m}, 1 \mathrm{H}), 7.42(\mathrm{t}, J=8.4 \mathrm{~Hz}, 1 \mathrm{H}), 7.27(\mathrm{~d}, J$ $=8.4 \mathrm{~Hz}, 1 \mathrm{H}), 3.24(\mathrm{sept}, J=6.8 \mathrm{~Hz}, 1 \mathrm{H}), 1.37(\mathrm{~d}, J=6.8 \mathrm{~Hz}$, $6 \mathrm{H}) \mathrm{ppm} .{ }^{13} \mathrm{C}$ NMR $\left(100 \mathrm{MHz}, \mathrm{CDCl}_{3}\right): \delta=167.4,147.6,136.2$, 129.0, 128.8, 127.2, 126.7, 125.4, 119.0, 37.1, 22.3. HRMS (ESITOF) calcd for $\mathrm{C}_{12} \mathrm{H}_{14} \mathrm{~N}(\mathrm{M}+\mathrm{H})^{+} \mathrm{m} / z=172.1126$, found 172.1134. These data are in agreement with the reported data.

\section{2-Phenylquinoline (12d) $)^{21 c}$}

Off-white solid (72 mg, 69\%). ${ }^{1} \mathrm{H}$ NMR $\left(400 \mathrm{MHz}, \mathrm{CDCl}_{3}\right): \delta=$ 8.20-8.14 (m, 4H), $7.85(\mathrm{~d}, J=8.8 \mathrm{~Hz}, 1 \mathrm{H}), 7.80(\mathrm{dd}, J=8.0$, $0.8 \mathrm{~Hz}, 1 \mathrm{H}), 7.73-7.69(\mathrm{~m}, 1 \mathrm{H}), 7.54-7.49(\mathrm{~m}, 3 \mathrm{H}), 7.47-7.43(\mathrm{~m}$, 1H) ppm. ${ }^{13} \mathrm{C}$ NMR $\left(100 \mathrm{MHz}, \mathrm{CDCl}_{3}\right): \delta=157.3,148.2,139.6$, $136.7,129.7,129.6,129.2,128.8,127.5,127.4,127.1,126.2$, $118.9 \mathrm{ppm}$. HRMS (ESI-TOF) calcd for $\mathrm{C}_{15} \mathrm{H}_{12} \mathrm{~N}(\mathrm{M}+\mathrm{H})^{+} \mathrm{m} / \mathrm{z}=$ 206.0970, found 206.0965. These data are in agreement with the reported data.

\section{Conflicts of interest}

There are no conflicts to declare.

\section{Acknowledgements}

We thank the Ministry of Science and Technology, Taiwan (Grant No. MOST106-2113-M-002-018) for the financial support. We also thank "National Taiwan University Mass Spectrometrybased Proteomics Core Facility" for the measurement of ESI mass data.

\section{Notes and references}

1 (a) S. Cretton, S. Dorsaz, A. Azzollini, Q. F. Godal, L. Marcourt, S. N. Ebrahimi, F. Voinesco, E. Michellod, D. Sanglard, M. Cuendet and P. Christen, J. Nat. Prod., 2016, 79, 300; (b) J. P. Michael, Nat. Prod. Rep., 2008, 25, 166; (c) J. P. Michael, Nat. Prod. Rep., 2004, 21, 650.

2 (a) O. Afzal, S. Kumar, M. R. Haider, M. R. Ali, R. Kumar, M. Jaggi and S. Bawa, Eur. J. Med. Chem., 2015, 97, 871; (b) S. Kumar, S. Bawa and H. Gupta, Mini-Rev. Med. Chem., 2009, 9, 1648; (c) D. J. Hazuda, P. Felock and M. Witmer, Science, 2000, 287, 646; (d) J. Wang, M. W. Chen, Y. Ji, S. B. Hu and Y. G. Zhou, J. Am. Chem. Soc., 2016, 138, 10413; (e) M. Heitbaum, R. Frchlich and F. Glorius, Adv. Synth. Catal., 2010, 352, 357, and references therein.

3 (a) L. A. Dawson, K. J. Cato, C. Scott, J. M. Watson, M. D. Wood, R. Foxton, R. De la Flor, G. A. Jones, J. N. Kew, J. E. Cluderay, E. Southam, G. S. Murkitt, J. Gartlon, D. J. Perberton, D. C. Jones, C. H. Davies and J. Hagen, Neuropsychopharmacology, 2008, 33, 1642; (b) P. W. Bedard, V. Clerin, N. Sushkova, B. Tchernychev, T. Antrilli, C. Resmini, J. C. Keith Jr, J. K. Hennan, N. Kaila, S. Debernardo, K. Janz, Q. Wang, D. L. Crandall, R. G. Schaub, G. D. Shaw and L. L. Carter, J. Pharmacol. Exp. Ther., 2008, 324, 497; (c) M. A. Massoud, S. A. El Bialy, W. A. Bayoumi and W. M. El Husseiny, Heterocycl. Commun., 2014, 20, 81.

4 V. Suchaud, F. Bailly and C. Lion, Bioorg. Med. Chem. Lett., 2012, 22, 3988.

5 Y. Aoki, M. Ishiwara, A. Koda and H. Takagaki, Eur. J. Pharmacol., 2000, 409, 325.

6 A. J. Duplantier, S. L. Becker and M. J. Bohanon, J. Med. Chem., 2009, 52, 3576.

7 S. A. Jenekhe, L. Lu and M. M. Alam, Macromolecules, 2001, 34, 7315 .

8 A. Heguy, P. Cai, P. Meyn, D. Houck, S. Russo, R. Michitsch, C. Pearce, B. Katz, G. Bringmann, D. Feineis, D. L. Taylor and A. S. Tyms, Antiviral Chem. Chemother., 1998, 9, 149.

9 D. L. Boger and J.-H. Chen, J. Org. Chem., 1995, 60, 7369.

10 (a) E. J. Cragoe, M. D. Bealor, C. M. Robb, C. Ziegler and J. M. Sprague, J. Org. Chem., 1953, 18, 561; (b) F. J. Kreysa, P. S. Forgione and V. F. Maturi, J. Org. Chem., 1955, 20, 971.

11 W. Zhang and J. M. Ready, Angew. Chem., Int. Ed., 2014, 53, 8980.

12 Y. Kobayashi and T. Harayama, Org. Lett., 2009, 11, 1603.

13 N. H. Cromwell and R. A. Setterquist, J. Am. Chem. Soc., 1954, 76, 5752 .

14 Z. He and T. F. Jamison, Angew. Chem., Int. Ed., 2014, 53, 3353.

15 P. Chauhan, M. Ravi, R. Kant and P. P. Yadav, Org. Biomol. Chem., 2017, 15, 1080.

16 V. A. Mamedov, V. L. Mamedova, V. V. Sykaev, D. E. Korshin, G. Z. Khikmatova, E. V. Mironova, O. B. Bazanova, L. K. Rizvanov and S. K. Latypova, Tetrahedron, 2017, 73, 5082 . 
17 S. W. Youn, H. J. Yoo, E. M. Lee and S. Y. Lee, Adv. Synth. Catal., 2018, 360, 278.

18 (a) M. Ramanathan and S.-T. Liu, Tetrahedron, 2017, 73, 4317; (b) M. Ramanathan and S.-T. Liu, J. Org. Chem., 2015, 80, 5329; (c) M. Ramanathan and S.-T. Liu, J. Org. Chem., 2017, 82, 8290.

19 M. Xie, X. Liu, Y. Zhu, X. Zhao, Y. Xia, L. Lin and X. Feng, Chem.-Eur. J., 2011, 17, 13800.

20 K. Janz and N. Kaila, J. Org. Chem., 2009, 74, 8874.
21 (a) X. Su, C. Chen, Y. Wang, J. Chen, Z. Lou and M. Li, Chem. Commun., 2013, 49, 6752; (b) J. I. Urzua, R. Contrera, C. O. Salasa and R. A Tapia, RSC Adv., 2016, 6, 82401; (c) K. H. He, F. F. Tan, C. Z. Zhou, G. J. Zhou, X. L. Yang and Y. Li, Angew. Chem., Int. Ed., 2017, 56, 3080; (d) N. T. Patil and V. S. Raut, J. Org. Chem., 2010, 75, 6961; (e) Y. F. Liang, X. F. Zhou, S. Y. Tang, Y. B. Huang, Y. S. Feng and H. J. Xu, RSC Adv., 2013, 3, 7739. 